\title{
The „Fingerprint” of the American Management in the Powerful Dynamics Concerning the Real G.D.P. from the United States of America
}

\author{
Gabriela OPAIT ${ }^{\star}$
}

\begin{tabular}{l}
\hline \multicolumn{1}{c}{ A R T I C L E I N F O } \\
\hline Article history: \\
Accepted April 2021 \\
Available online May 2021 \\
\hline JEL Classification \\
C1, C12, C2 \\
Keywords: \\
American management, American \\
smart style, Real G.D.P., Annual \\
growth \\
\hline
\end{tabular}

\begin{abstract}
A B S T R A C T
The victorious spirit, which predominates in all the provinces of the United States of America, penetrates the scale of values in rise concerning the real G.D.P. The symbiosis of the progresses witnessed by the American nation along of the time, as a result of the management organized in "American smart style", have reflection in the values regarding the real G.D.P. of the United States of America. The aim of this original research pursues to display the permanent increase regarding the real G.D.P. of the United States of America, between 2021-2030.
\end{abstract}

(C) 2021 EAI. All rights reserved.

\section{Introduction}

The American management represents a real brand, a true star in the relatively speaking worldwide management and this phenomenon is a positive vector for the towering standard of the life in the United states of America. The pulsations concerning the powerful vibrations of the American management in all the economic and social spheres have as effects augmentations of the real G.D.P. in the United States of America. The objective of this original research is indicated by the representation of the real G.D.P.'s rises which will be in the United States of America, between 2021-2030, as effects of the american management's performances. In the first episode of this research, we can see the proceeding' s steps which have as purpose the display of the "radiography" for the statistical data which show us the „wellness” regarding the real G.D.P. in the United States of America, between 2010-2020, „wellness in mirroring” with the period 2021-2030. In the second episode of this article, we can observe the proceeding' s steps which follow the diagnostic regarding the "wellness" concerning the annual growth of the real G.D.P. in the United States of America, between 2010 2019, in corelation with the same „wellness” projected between 2021-2030. The both episodes, the third and the fourth of this paper, achieve the „polarization” concerning the steps which have within sight the statistical values between 2010-2018, concerning the G.D.P. per capita in PPP, respectively the G.D.P. per capita in PPS for the United States of America, for "to mirror" them wellness' diagnostics in the period 2021-2030, respectively in 2021. In top management of the methods applied for these projections, which have in background actions for to „X-ray” the G,D.P.'s „wellness” in the United States, lead the „Least Squares Method” and the forecast's method. The past master who shaped the structure of the "Least Squares Method" which is the „best ingredient” for to mould forecasts, was Johann Carl Friedrich Gauss.

2. The proceeding' s steps which have as aim the representation for the trend model concerning the statistical data of the real G.D.P. in United States of America, in the period 2010-2020

Table 1. The real G.D.P. in the United States of America, between 2010-2020

\begin{tabular}{|c|c|}
\hline YEARS & $\begin{array}{c}\text { THE REAL G.D.P. IN THE UNITED STATES OF AMERICA } \\
\text { (\$ billions chained 2012) }\left(\xi_{i}\right)\end{array}$ \\
\hline 2010 & 15598,8 \\
\hline 2011 & 15840,7 \\
\hline 2012 & 16197,0 \\
\hline 2013 & 16495,4 \\
\hline 2014 & 16912,0 \\
\hline 2016 & 17432,2 \\
\hline
\end{tabular}




\begin{tabular}{|c|c|}
\hline YEARS & $\begin{array}{c}\text { THE REAL G.D.P. IN THE UNITED STATES OF AMERICA } \\
\text { (\$ billions chained 2012) }\left(\xi_{i}\right)\end{array}$ \\
\hline 2017 & $\mathbf{1 8 1 4 4 , 1}$ \\
\hline 2018 & $\mathbf{1 8 6 8 7 , 8}$ \\
\hline 2019 & $\mathbf{1 9 0 9 1 , 7}$ \\
\hline 2020 & $\mathbf{1 8 4 2 2 , 6}$ \\
\hline \multicolumn{2}{|c|}{ Source: „Statista Portal United States of America” }
\end{tabular}

- if the proceeding's structure, which draws up the trend for the $\xi$ variable, where $\xi=$ the real G.D.P. in United States, selects a linear exposure $\xi_{t_{i}}=a+b \cdot t_{i}, a$ and $b$ will be [4]:

$$
a=\frac{\left|\begin{array}{ll}
\sum_{i=1}^{n} \xi_{i} & \sum_{i=1}^{n} t_{i} \\
\sum_{i=1}^{n} \xi_{i} t_{i} & \sum_{i=1}^{n} t_{i}{ }^{2}
\end{array}\right|}{\left|\begin{array}{cc}
n & \sum_{i=1}^{n} t_{i} \\
\sum_{i=1}^{n} t_{i} & \sum_{i=1}^{n} t_{i}{ }^{2}
\end{array}\right|}=\frac{\sum_{i=1}^{n} \xi_{i} \sum_{i=1}^{n} t_{i}{ }^{2}-\sum_{i=1}^{n} \xi_{i} t_{i} \sum_{i=1}^{n} t_{i}}{n \sum_{i=1}^{n} t_{i}{ }^{2}-\left(\sum_{i=1}^{n} t_{i}\right)^{2}} \quad b=\frac{\left|\begin{array}{cc}
n & \sum_{i=1}^{n} x_{i} \\
\sum_{i=1}^{n} t_{i} & \sum_{i=1}^{n} \xi_{i} t_{i}
\end{array}\right|}{\left|\begin{array}{cc}
n & \sum_{i=1}^{n} t_{i} \\
\sum_{i=1}^{n} t_{i} & \sum_{i=1}^{n} t_{i}{ }^{2}
\end{array}\right|}=\frac{n \sum_{i=1}^{n} \xi_{i} t_{i}-\sum_{i=1}^{n} t_{i} \sum_{i=1}^{n} \xi_{i}}{n \sum_{i=1}^{n} t_{i}{ }^{2}-\left(\sum_{i=1}^{n} t_{i}\right)^{2}}
$$

Table 2. The „radiography” for the values of the real G.D.P. in the United States,

\begin{tabular}{|c|c|c|c|c|c|c|}
\hline \multirow{2}{*}{ YEARS } & \multirow{2}{*}{$\begin{array}{c}\text { THE REAL G.D.P. } \\
\text { IN THE UNITED } \\
\text { STATES OF AMERICA } \\
\text { (\$ billions chained } \\
\text { 2012) } \\
\left(\xi_{i}\right) \\
\end{array}$} & \multicolumn{5}{|c|}{ LINEAR TENDENCY } \\
\hline & & $t_{i}$ & $t_{i}^{2}$ & $t_{i} \xi_{i}$ & $\xi_{t_{i}}=a+b t_{i}$ & $\mid \xi_{i}-\xi_{t_{i}}$ \\
\hline 2012 & 16197,0 & -3 & 9 & $-48591,0$ & 16267,21909 & 70,2 \\
\hline 2013 & 16495,4 & -2 & 4 & $-32990,8$ & 16619,14000 & 123,7 \\
\hline 2014 & 16912,0 & -1 & 1 & $-16912,0$ & 16971,06091 & 59,1 \\
\hline 2015 & 17432,2 & 0 & 0 & 0 & 17322,98182 & 109,2 \\
\hline 2016 & 17730,5 & +1 & 1 & $+17730,5$ & 17674,90273 & 55,6 \\
\hline 2020 & 18422,6 & +5 & 25 & $+92113,0$ & 19082,58637 & 660,0 \\
\hline TOTAL & 190552,8 & 0 & 110 & 38711,3 & & 1975,2 \\
\hline
\end{tabular}

$$
\begin{gathered}
a=\frac{190552,8 \cdot 110}{11 \cdot 110}=17322,98182 \quad b=\frac{11 \cdot 38711,3}{11 \cdot 110}=351,9209091 \\
v_{I}=\left[\frac{\sum_{i=1}^{m}\left|\xi_{i}-\xi_{t_{i}}^{I}\right|}{n}: \frac{\sum_{i=1}^{m} \xi_{i}}{n}\right] \cdot 100=\frac{\sum_{i=1}^{m}\left|\xi_{i}-\xi_{t_{i}}\right|}{\sum_{i=1}^{m} \xi_{i}} \cdot 100=\frac{1975,2}{190552,8} \cdot 100=1,04 \%
\end{gathered}
$$

- if the proceeding's structure which draws up the trend of the modeling for $\xi$ variable, where $\xi=$ the real G.D.P. in United States, selects a parabolic exposure $\xi_{t_{i}}=a+b \cdot t_{i}+c t_{i}^{2}, a$ and $b$ will be [4]:

Table 3. The „radiography” for the values of the real G.D.P. in the United States,

\begin{tabular}{|c|c|c|c|c|c|c|c|c|}
\hline \multirow{2}{*}{ if this indicates a quadratic exposure } \\
\hline & $\begin{array}{c}\text { THE REAL G.D.P. } \\
\text { IN THE UNITED STATES } \\
\text { OF AMERICA } \\
\text { YEARS }\end{array}$ & \multicolumn{7}{|c|}{ PARABOLIC TENDENCY } \\
\cline { 3 - 9 } & $\begin{array}{c}\text { \$ billions chained 2012) } \\
\left(\xi_{i}\right)\end{array}$ & $t_{i}$ & $t_{i}^{2}$ & $t_{i}^{3}$ & $t_{i}^{4}$ & $t_{i}^{2} \xi_{i}$ & $\xi_{t_{i}}=a+b \xi_{i}+c \xi_{i}^{2}$ & $\left|\xi_{i}-\xi_{t_{i}}\right|$ \\
\hline $\mathbf{2 0 1 0}$ & $\mathbf{1 5 5 9 8 , 8}$ & -5 & 25 & -125 & 625 & 389970,0 & 15407,59231 & 191,2 \\
\hline $\mathbf{2 0 1 1}$ & $\mathbf{1 5 8 4 0 , 7}$ & -4 & 16 & -64 & 256 & 253451,2 & 15852,98419 & 12,3 \\
\hline $\mathbf{2 0 1 2}$ & $\mathbf{1 6 1 9 7 , 0}$ & -3 & 9 & -27 & 81 & 145773,0 & 16277,60475 & 80,6 \\
\hline
\end{tabular}




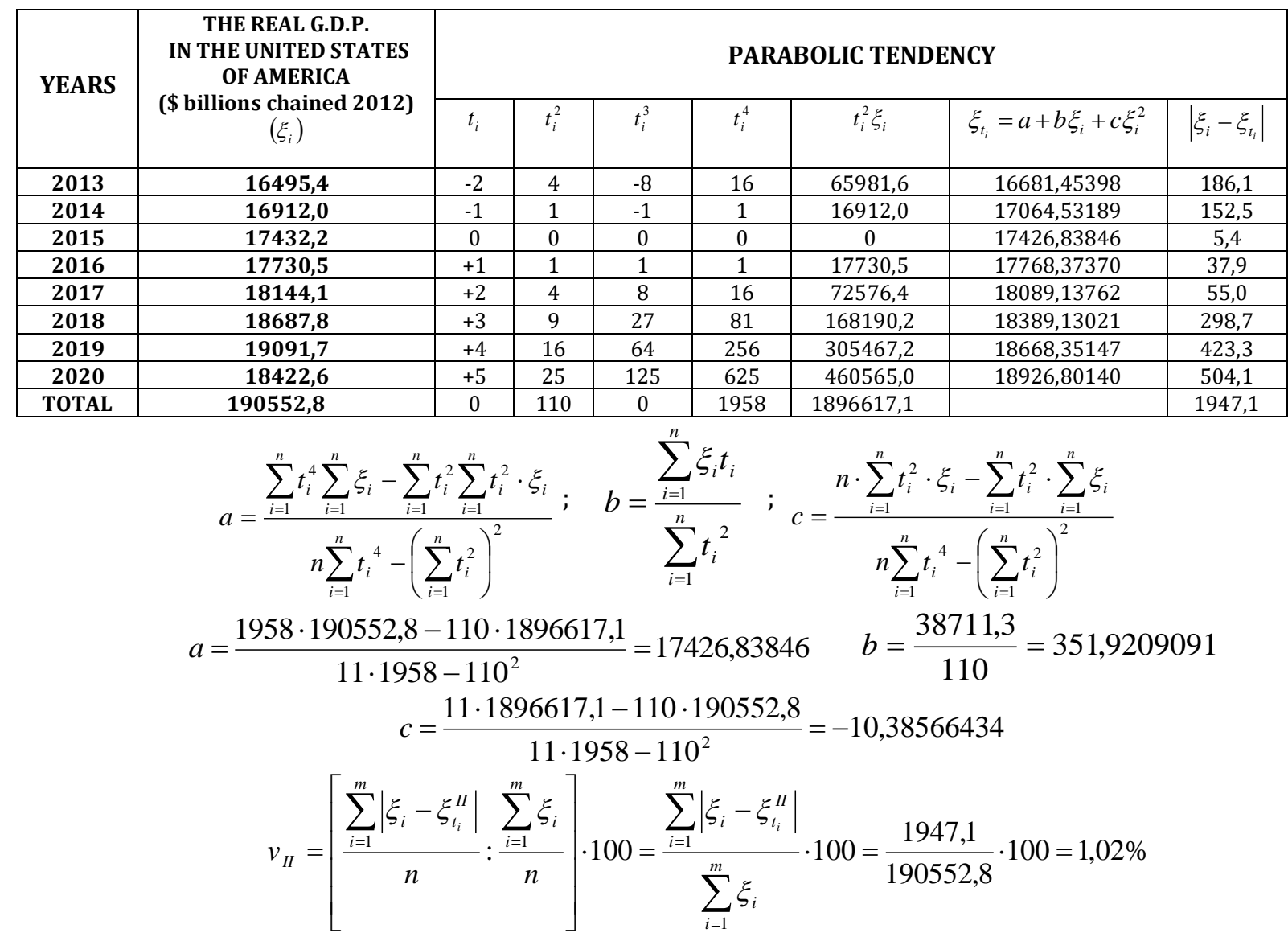

- if the proceeding's structure which draws up the trend for $\xi$ variable, where $\xi=$ the real G.D.P. in United States, selects an exponential exposure $\xi_{t_{i}}=a b^{t_{i}}, a$ and $b$ will be [4]:

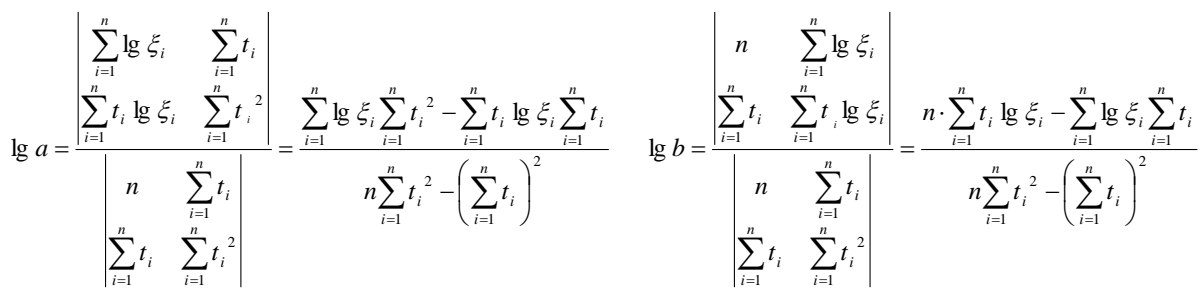

Table 4. The „radiography” for the values of the real G.D.P. in the United States, if this indicates an exponential exposure

\begin{tabular}{|c|c|c|c|c|c|c|c|}
\hline \multirow{2}{*}{ YEARS } & \multirow{2}{*}{$\begin{array}{c}\text { THE REAL G.D.P. } \\
\text { IN THE UNITED } \\
\text { STATES OF AMERICA } \\
\text { (\$ billions chained 2012) } \\
\left(\xi_{i}\right)\end{array}$} & \multicolumn{6}{|c|}{ EXPONENTIAL TENDENCY } \\
\hline & & $t_{i}$ & $\lg \xi_{i}$ & $t_{i} \lg \xi_{i}$ & $\lg \xi_{t_{i}}=\lg a+t_{i} \lg b$ & $\xi_{t_{i}}=a b^{t_{i}}$ & $\left|\xi_{i}-\xi_{t_{i}}\right|$ \\
\hline 2010 & 15598,8 & -5 & 4,193091190 & $-20,96545595$ & 4,193346321 & 15607,96638 & 9,2 \\
\hline 2011 & 15840,7 & -4 & 4,199774369 & $-16,79909748$ & 4,202211971 & 15929,86044 & 89,2 \\
\hline 2012 & 16197,0 & -3 & 4,209434582 & $-12,62830375$ & 4,211077621 & 16258,39314 & 61,4 \\
\hline 2013 & 16495,4 & -2 & 4,217362851 & $-8,434725703$ & 4,219943271 & 16593,70141 & 98,3 \\
\hline 2014 & 16912,0 & -1 & 4,228194970 & $-4,228194970$ & 4,228808921 & 16935,92497 & 23,9 \\
\hline 2015 & 17432,2 & 0 & 4,241352200 & 0 & 4,237674571 & 17285,20645 & 147,0 \\
\hline 2016 & 17730,5 & +1 & 4,248720983 & 4,248720983 & 4,246540221 & 17641,69141 & 88,8 \\
\hline 2017 & 18144,1 & +2 & 4,258735431 & 8,517470862 & 4,255405871 & 18005,52840 & 138,6 \\
\hline 2018 & 18687,8 & +3 & 4,271558178 & 12,81467453 & 4,264271521 & 18376,86907 & 310,9 \\
\hline 2019 & 19091,7 & +4 & 4,280844601 & 17,12337841 & 4,273137171 & 18755,86815 & 335,8 \\
\hline 2020 & 18422,6 & +5 & 4,265350923 & 21,32675461 & 4,282002821 & 19142,68359 & 720,1 \\
\hline TOTAL & 190552,8 & 0 & 46,61442028 & 0,975221545 & & & 2023,2 \\
\hline
\end{tabular}




$$
\begin{gathered}
\lg a=\frac{46,61442028 \cdot 110}{11 \cdot 110}=4,237674571 \quad \lg b=\frac{11 \cdot 0,975221545}{11 \cdot 110}=0,00886565 \\
v_{\text {exp }}=\left[\frac{\sum_{i=1}^{n}\left|\xi_{i}-\xi_{t_{i}}^{\exp }\right|}{n}: \frac{\sum_{i=1}^{n} \xi_{i}}{n}\right] \cdot 100=\frac{\sum_{i=1}^{n}\left|\xi_{i}-\xi_{t_{i}}^{\text {exp }}\right|}{\sum_{i=1}^{n} \xi_{i}} \cdot 100=\frac{2023,2}{190552,8} \cdot 100=1,06 \% \\
v_{I I}=1,02 \%<v_{I}=1,04 \%<v_{\text {exp }}=1,06 \%
\end{gathered}
$$

The values concerning the real G.D.P. in the United States of America, between 2010-2020, touch a quadratic "target” $\xi_{t_{i}}=a+b \cdot t_{i}+c t_{i}^{2}$.

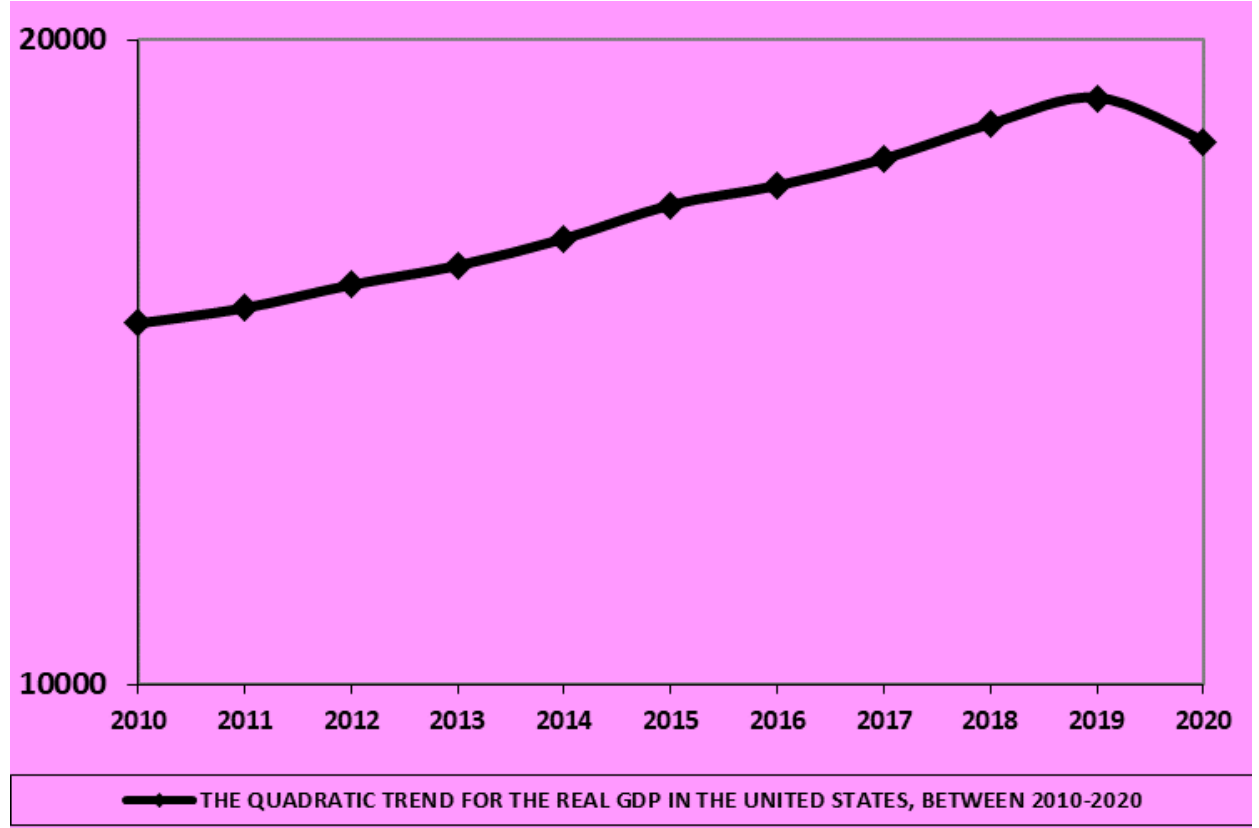

Graph 1. The quadratic „radiography” of the statistical data which show us the "wellness" regarding the real G.D.P. in the United States of America, between 2010-2020

$\xi_{2021}^{R E A L_{-} G D P}=17426,83846+351,9209091 \cdot 6+(-10,38566434) \cdot 6^{2}=\$ 19164,5 \_$billions
$\xi_{2022}^{R E A L_{-} G D P}=17426,83846+351,9209091 \cdot 7+(-10,38566434) \cdot 7^{2}=\$ 19381,4 \_$billions
$\xi_{2023}^{R E A L_{-} G D P}=17426,83846+351,9209091 \cdot 8+(-10,38566434) \cdot 8^{2}=\$ 19577,5 \_$billions
$\xi_{2024}^{R E A L_{-} G D P}=17426,83846+351,9209091 \cdot 9+(-10,38566434) \cdot 9^{2}=\$ 19752,9 \_$billions
$\xi_{2025}^{R E A L_{-} G D P}=17426,83846+351,9209091 \cdot 10+(-10,38566434) \cdot 10^{2}=\$ 19907,5 \_$billions
$\xi_{2026}^{R E A L_{-} G D P}=17426,83846+351,9209091 \cdot 11+(-10,38566434) \cdot 11^{2}=\$ 20041,3 \_$billions
$\xi_{2027}^{R E A L_{-} G D P}=17426,83846+351,9209091 \cdot 12+(-10,38566434) \cdot 12^{2}=\$ 20154,4 \_$billions
$\xi_{2028}^{R E A L_{-} G D P}=17426,83846+351,9209091 \cdot 13+(-10,38566434) \cdot 13^{2}=\$ 20246,6 \_$billions
$\xi_{2029}^{R E A L_{-} G D P}=17426,83846+351,9209091 \cdot 14+(-10,38566434) \cdot 14^{2}=\$ 20318,1$ billions
$\xi_{2030}^{R E A L_{-} G D P}=17426,83846+351,9209091 \cdot 15+(-10,38566434) \cdot 15^{2}=\$ 20368,9 \_$billions 
3. The proceeding' s steps, which pursue the statistical data regarding the annual growth of the Real G.D.P. in United States of America, for to achieve them wellness' diagnostic

Table 5. The annual growth of the real G.D.P. in the United States, between 2010-2019

\begin{tabular}{|c|c|}
\hline YEARS & $\begin{array}{c}\text { THE ANNUAL GROWTH OF THE REAL G.D.P. } \\
\text { IN THE UNITED STATES OF AMERICA } \\
(\%) \quad\left(\lambda_{i}\right)\end{array}$ \\
\hline 2010 & 2,6 \\
\hline 2011 & 1,6 \\
\hline 2012 & 2,2 \\
\hline 2013 & 1,8 \\
\hline 2014 & 2,5 \\
\hline 2015 & 2,9 \\
\hline 2017 & 1,6 \\
\hline 2018 & 2,4 \\
\hline 2019 & 2,9 \\
\hline
\end{tabular}

- if the proceeding's structure which draws up the trend for the $\lambda$ variable, where $\lambda=$ the annual growth of the Real G.D.P. in United States, selects a linear exposure $\lambda_{t_{i}}=a+b \cdot t_{i}, a$ and $b$ will be [4]:

Table 6. The „radiography” for the annual growth's values concerning the real G.D.P. in the United States, if this indicates a linear exposure

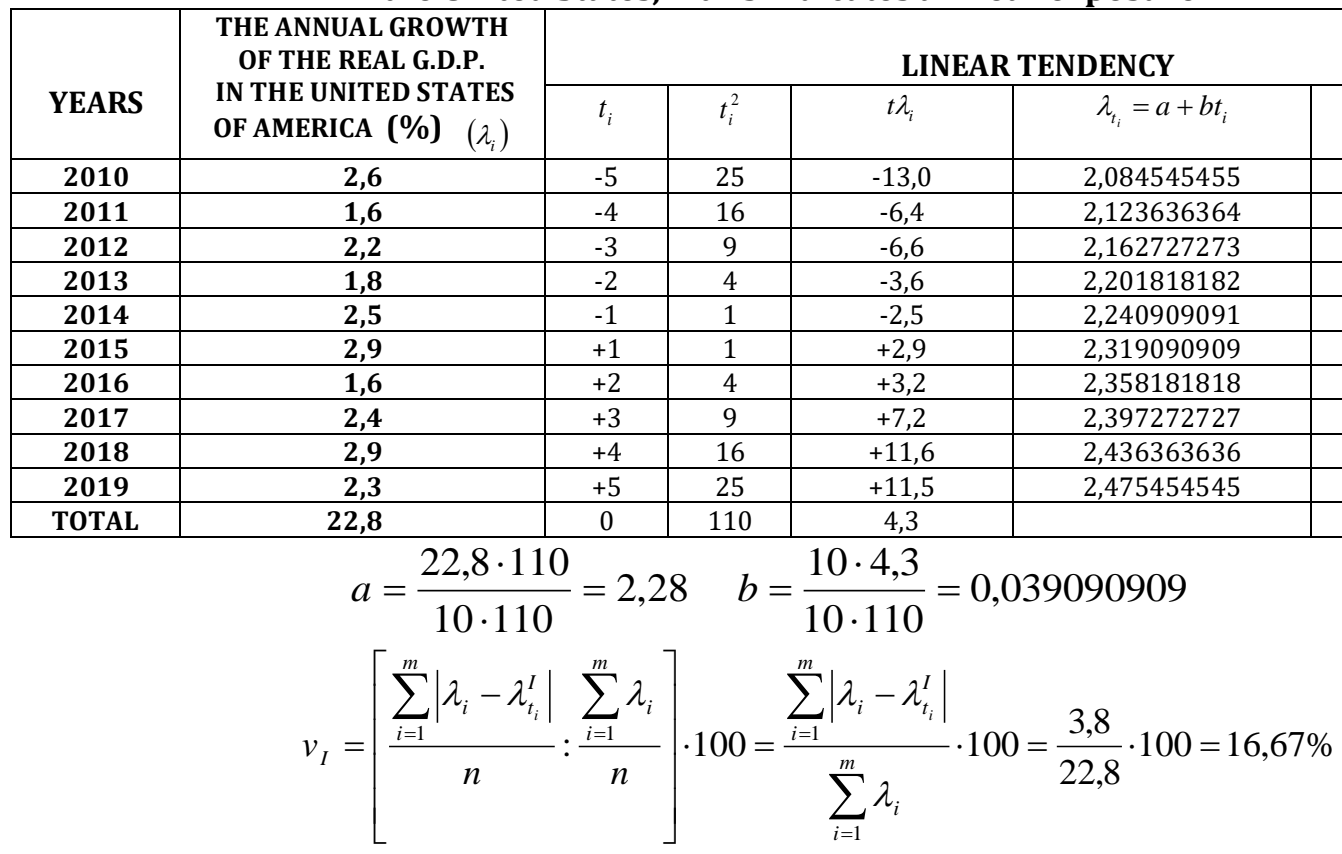

- if the proceeding's structure which draws up the trend for $\lambda$ variable, where $\lambda=$ the annual growth of the Real G.D.P. in United States, selects a parabolic exposure $\lambda_{t_{i}}=a+b \cdot t_{i}+c t_{i}^{2}, a$ and $b$ will be [4]:

Table 7. The „radiography" for the annual growth's values regarding the real G.D.P. in the United States, if this indicates a quadratic exposure

\begin{tabular}{|c|c|c|c|c|c|c|c|c|}
\hline \multirow{2}{*}{ YEARS } & \multirow{2}{*}{$\begin{array}{c}\text { THE ANNUAL } \\
\text { GROWTH OF THE } \\
\text { REAL G.D.P. } \\
\text { IN THE UNITED } \\
\text { STATES OF AMERICA } \\
(\%)\left(\lambda_{i}\right)\end{array}$} & \multicolumn{7}{|c|}{ PARABOLIC TENDENCY } \\
\hline & & $t_{i}$ & $t_{i}^{2}$ & $t_{i}^{3}$ & $t_{i}^{4}$ & $t_{i}^{2} \lambda_{i}$ & $\lambda_{t_{i}}=a+b \lambda_{i}+c \lambda_{i}^{2}$ & $\left|\lambda_{i}-\lambda_{t_{i}}\right|$ \\
\hline 2010 & 2,6 & -5 & 25 & -125 & 625 & 65,0 & 2,161283423 & 0,4 \\
\hline 2011 & 1,6 & -4 & 16 & $\begin{array}{l}-64 \\
\end{array}$ & 256 & 25,6 & 2,151042774 & 0,5 \\
\hline 2012 & 2,2 & -3 & 9 & -27 & 81 & 19,8 & 2,151764702 & 0,1 \\
\hline 2013 & 1,8 & -2 & 4 & -8 & 16 & 7,2 & 2,163449196 & 0,4 \\
\hline 2014 & 2,5 & -1 & 1 & -1 & 1 & 2,5 & 2,186096256 & 0,3 \\
\hline 2015 & 2,9 & +1 & 1 & +1 & 1 & 2,9 & 2,264278074 & 0,6 \\
\hline 2016 & 1,6 & +2 & 4 & +8 & 16 & 6,4 & 2,319812832 & 0,7 \\
\hline 2017 & 2,4 & +3 & 9 & +27 & 81 & 21,6 & 2,386310156 & 0 \\
\hline
\end{tabular}




\begin{tabular}{|c|c|c|c|c|c|c|c|c|}
\hline \multirow{2}{*}{ YEARS } & \multirow{2}{*}{$\begin{array}{c}\text { THE ANNUAL } \\
\text { GROWTH OF THE } \\
\text { REAL G.D.P. } \\
\text { IN THE UNITED } \\
\text { STATES OF AMERICA } \\
(\%)\left(\lambda_{i}\right)\end{array}$} & \multicolumn{7}{|c|}{ PARABOLIC TENDENCY } \\
\hline & & $t_{i}$ & $t_{i}^{2}$ & $t_{i}^{3}$ & $t_{i}^{4}$ & $t_{i}^{2} \lambda_{i}$ & $\lambda_{t_{i}}=a+b \lambda_{i}+c \lambda_{i}^{2}$ & $\mid \lambda_{i}-\lambda_{t_{i}}$ \\
\hline 2018 & 2,9 & +4 & 16 & +64 & 256 & 46,4 & 2,463784446 & 0,4 \\
\hline 2019 & 2,3 & +5 & 25 & +125 & 625 & 57,5 & 2,552192502 & 0,3 \\
\hline TOTAL & 22,8 & 0 & 110 & 0 & 1958 & 254,9 & & 3,7 \\
\hline
\end{tabular}

$$
\begin{gathered}
a=\frac{1958 \cdot 22,8-110 \cdot 254,9}{10 \cdot 1958-110^{2}}=2,219705882 \quad b=\frac{4,3}{110}=0,039090909 \\
c=\frac{10 \cdot 254,9-110 \cdot 22,8}{10 \cdot 1958-110^{2}}=0,005481283 \\
v_{I I}=\left[\frac{\sum_{i=1}^{m}\left|\lambda_{i}-\lambda_{t_{i}}^{I I}\right|}{n}: \frac{\sum_{i=1}^{m} \lambda_{i}}{n}\right] \cdot 100=\frac{\sum_{i=1}^{m}\left|\lambda_{i}-\lambda_{t_{i}}^{I I}\right|}{\sum_{i=1}^{m} \lambda_{i}} \cdot 100=\frac{3,7}{22,8} \cdot 100=16,23 \%
\end{gathered}
$$

- if the proceeding's structure which draws up the trend for $\lambda$ variable, where $\lambda=$ the annual growth of the Real G.D.P. in United States, selects an exponential exposure $\lambda_{t_{i}}=a b^{t_{i}}, a$ and $b$ will be [4]:

\begin{tabular}{|c|c|c|c|c|c|c|c|}
\hline \multirow{2}{*}{ YEARS } & \multirow{2}{*}{$\begin{array}{c}\text { THE ANNUAL } \\
\text { GROWTH OF THE } \\
\text { REAL G.D.P. } \\
\text { IN THE UNITED } \\
\text { STATES OF } \\
\text { AMERICA (\%) }\left(\lambda_{i}\right)\end{array}$} & \multicolumn{6}{|c|}{ EXPONENTIAL TENDENCY } \\
\hline & & $t_{i}$ & $\lg \lambda_{i}$ & $t_{i} \lg \lambda_{i}$ & $\lg \lambda_{t_{i}}=\lg a+t_{i} \lg b$ & $\lambda_{t_{i}}=a b^{t_{i}}$ & $\left|\lambda_{i}-\lambda_{t_{i}}\right|$ \\
\hline 2010 & 2,6 & -5 & 0,414973348 & $-2,074866740$ & 0,310267307 & 2,042995015 & 0,6 \\
\hline 2011 & 1,6 & -4 & 0,204119982 & $-0,816479930$ & 0,317925517 & 2,079340043 & 0,5 \\
\hline 2012 & 2,2 & -3 & 0,342422680 & $-1,027268042$ & 0,325583727 & 2,116331650 & 0,1 \\
\hline 2013 & 1,8 & -2 & 0,255272505 & $-0,510545010$ & 0,333241937 & 2,153981342 & 0,4 \\
\hline 2014 & 2,5 & -1 & 0,397940008 & $-0,397940008$ & 0,340900147 & 2,192300824 & 0,3 \\
\hline 2015 & 2,9 & +1 & 0,462397997 & $+0,462397997$ & 0,356216567 & 2,270997034 & 0,6 \\
\hline 2016 & 1,6 & +2 & 0,204119982 & $+0,408239965$ & 0,363874777 & 2,311398233 & 0,7 \\
\hline 2017 & 2,4 & +3 & 0,380211241 & $+1,140633725$ & 0,371532987 & 2,352518173 & 0,1 \\
\hline 2018 & 2,9 & +4 & 0,462397997 & $+1,849591992$ & 0,379191197 & 2,394369639 & 0,5 \\
\hline 2019 & 2,3 & +5 & 0,361727836 & $+1,808639180$ & 0,386849407 & 2,436965646 & 0,1 \\
\hline TOTAL & 22,8 & 0 & 3,485583576 & 0,842403129 & & & 3,9 \\
\hline
\end{tabular}

Table 8. The „radiography” for the annual growth's values concerning the real G.D.P. in the United States, if this indicates an exponential exposure

$$
\begin{aligned}
& \lg a=\frac{3,485583576 \cdot 110}{10 \cdot 110}=0,348558357 \quad \lg b=\frac{10 \cdot 0,842403129}{10 \cdot 110}=0,00765821 \\
& v_{\text {exp }}=\left[\frac{\sum_{i=1}^{n}\left|\lambda_{i}-\lambda_{t_{i}}^{\text {exp }}\right|}{n}: \frac{\sum_{i=1}^{n} \lambda_{i}}{n}\right] \cdot 100=\frac{\sum_{i=1}^{n}\left|\lambda_{i}-\lambda_{t_{i}}^{\text {exp }}\right|}{\sum_{i=1}^{n} \lambda_{i}} \cdot 100=\frac{3,9}{22,8} \cdot 100=17,11 \% \\
& v_{I I}=16,23 \%<v_{I}=16,67 \%<v_{\text {exp }}=17,11 \%
\end{aligned}
$$

The values regarding the annual growth of the real G.D.P. in United States of America, in the period 20102019, hit a quadratic "target” $\lambda_{t_{i}}=a+b \cdot t_{i}+c t_{i}^{2}$. 


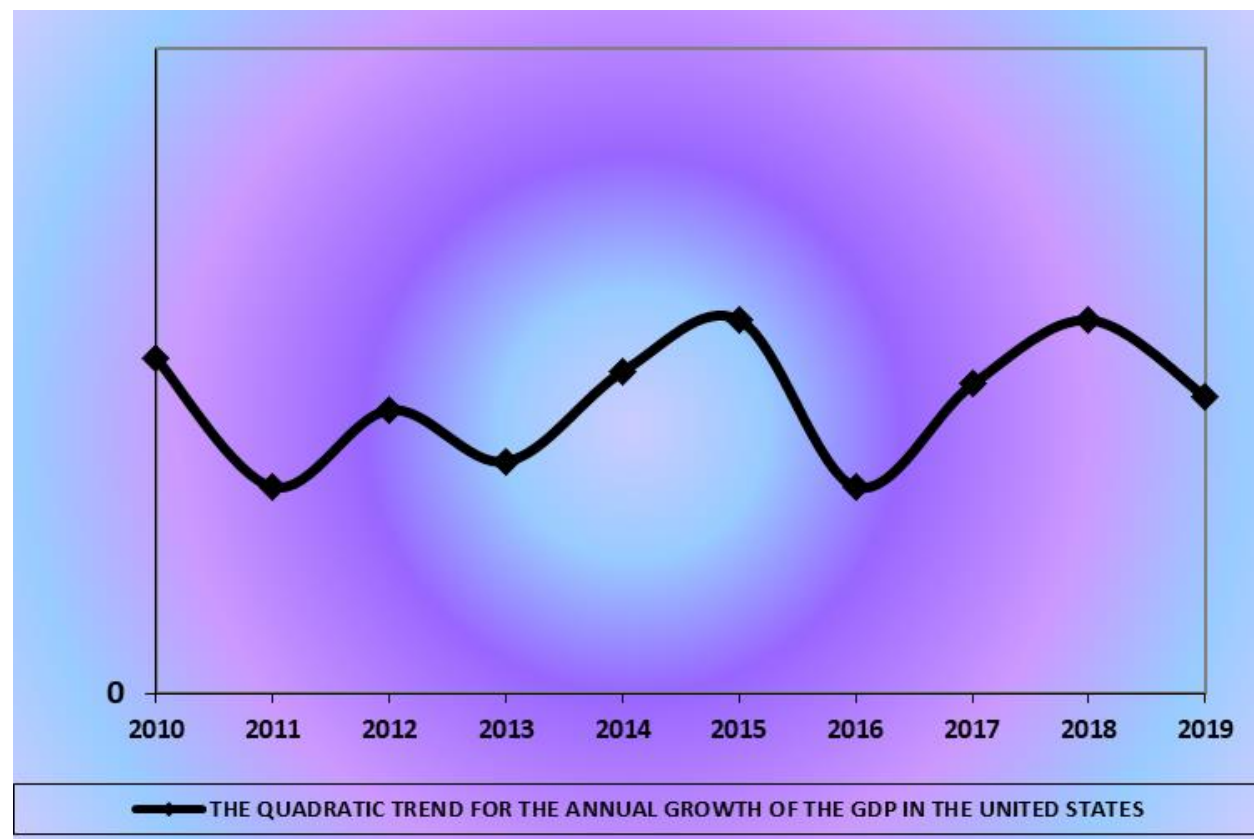

Graph 2. The quadratic "radiography” of the statistical data which show us the „wellness" concerning the annual growth of the Real G.D.P. in the United States of America, between 2010-2019

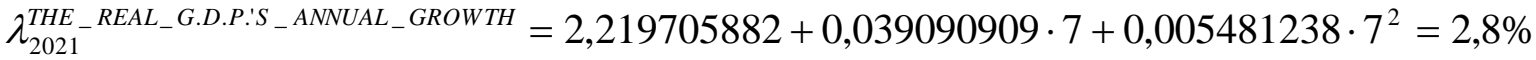

$\lambda_{2022}^{\text {THE }}{ }_{-}$REAL_G.D.P.'S ANNUAL_GROWTH $_{-}=2,219705882+0,039090909 \cdot 8+0,005481238 \cdot 8^{2}=2,9 \%$

$\lambda_{2023}^{\text {THE }}{ }_{-}$EAL_G.D.P.' $S_{-}$ANNUAL_GROWTH $=2,219705882+0,039090909 \cdot 9+0,005481238 \cdot 9^{2}=3,0 \%$

$\lambda_{2024}^{\text {THE }}$ REAL_G.D.P.'S $_{-}$ANNUAL_GROWTH $=2,219705882+0,039090909 \cdot 10+0,005481238 \cdot 10^{2}=3,2 \%$

$\lambda_{2025}^{\text {THE }}{ }^{\text {REAL_G.D.P.'S }}$ ANNUAL_GROWTH $_{-}=2,219705882+0,039090909 \cdot 11+0,005481238 \cdot 11^{2}=3,3 \%$

$\lambda_{2026}^{\text {THE }}$ REAL_G.D.P.'S $_{-}$ANNUAL_GROWTH $=2,219705882+0,039090909 \cdot 12+0,005481238 \cdot 12^{2}=3,5 \%$

$\lambda_{2027}^{\text {THE }}$ REAL_G.D.P.'S $_{-}$ANNUAL_GROWTH $=2,219705882+0,039090909 \cdot 13+0,005481238 \cdot 13^{2}=3,7 \%$

$\lambda_{2028}^{\text {THE }}{ }_{-}$REAL_G.D.P.'S S ANNUAL_GROWTH $_{-}=2,219705882+0,039090909 \cdot 14+0,005481238 \cdot 14^{2}=3,8 \%$

$\lambda_{2029}^{\text {THE }}{\text { REAL_G.D.P.'S } S_{-} \text {ANNUAL_GROWTH }}^{2}=2,219705882+0,039090909 \cdot 15+0,005481238 \cdot 15^{2}=4,0 \%$

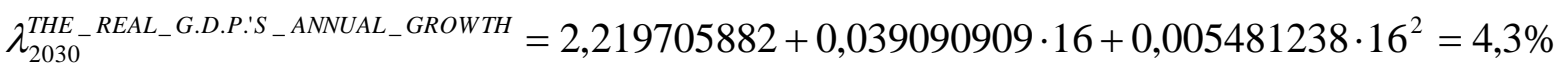

3. The proceeding' s steps, which follow the statistical values concerning the G.D.P. per capita in PPP for the United States of America, for to achieve them wellness' diagnostic

Table 9 The G.D.P. per capita in PPP's configuration in the United States of America, between 2010-2018

\begin{tabular}{|c|c|}
\hline \multirow{2}{*}{ YEARS } & $\begin{array}{c}\text { G.D.P. PER CAPITA IN PPP } \\
\text { THE UNITED STATES OF AMERICA } \\
\text { (\$) }\left(\omega_{i}\right)\end{array}$ \\
\hline 2010 & 49479,25 \\
\hline 2011 & 49883,11 \\
\hline 2012 & 50632,44 \\
\hline 2013 & 51208,89 \\
\hline 2014 & 52080,79 \\
\hline 2015 & 53187,57 \\
\hline 2016 & 53631,76 \\
\hline 2018 & 54470,80 \\
\hline
\end{tabular}

Source: „Trade Economics”

- if the proceeding's structure which draws up the trend for $\omega$ variable, where $\omega=$ G.D.P. per capita in PPP for the United States of America, selects a linear exposure $\omega_{t_{i}}=a+b \cdot t_{i}, a$ and $b$ will be [4]: 
Table 10. The "radiography" for the values of the G.D.P. per capita in PPP, in the United States of America, if this indicates a linear exposure

\begin{tabular}{|c|c|c|c|c|c|c|}
\hline \multirow{2}{*}{ YEARS } & \multirow{2}{*}{$\begin{array}{l}\text { G.D.P. PER CAPITA IN PPP } \\
\text { THE UNITED STATES } \\
\text { (\$) } \\
\left(\omega_{i}\right)\end{array}$} & \multicolumn{5}{|c|}{ LINEAR TENDENCY } \\
\hline & & $t_{i}$ & $t_{i}^{2}$ & $t_{i} \omega_{i}$ & $\omega_{t_{i}}=a+b t_{i}$ & $\left|\omega_{i}-\omega_{t_{i}}\right|$ \\
\hline 2010 & 49479,25 & -4 & 16 & $-197917,00$ & 49141,53422 & 337,72 \\
\hline 2011 & 49883,11 & -3 & 9 & $-149649,33$ & 49919,86539 & 36,75 \\
\hline 2012 & 50632,44 & -2 & 4 & $-101264,88$ & 50698,19656 & 65,76 \\
\hline 2013 & 51208,89 & -1 & 1 & $-51208,89$ & 51476,52772 & 267,64 \\
\hline 2014 & 52080,79 & 0 & 0 & 0 & 52254,85889 & 174,07 \\
\hline 2015 & 53187,57 & +1 & 1 & $+53187,57$ & 53033,19006 & 154,38 \\
\hline 2016 & 53631,76 & +2 & 4 & $+107263,52$ & 53811,52122 & 179,76 \\
\hline 2017 & 54470,80 & +3 & 9 & $+163412,40$ & 54589,85239 & 119,05 \\
\hline 2018 & 55719,12 & +4 & 16 & $+222876,48$ & 55368,18356 & 350,94 \\
\hline TOTAL & 470293,73 & 0 & 60 & 46699,87 & & 1686,07 \\
\hline
\end{tabular}

$$
\begin{gathered}
a=\frac{470293,73 \cdot 60}{9 \cdot 60}=52254,85889 \quad b=\frac{9 \cdot 46699,87}{9 \cdot 60}=778,3311667 \\
v_{I}=\left[\frac{\sum_{i=1}^{n}\left|\omega_{i}-\omega_{t_{i}}^{I}\right|}{n}: \frac{\sum_{i=1}^{n} \omega_{i}}{n}\right] \cdot 100=\frac{\sum_{i=1}^{n}\left|\omega_{i}-\omega_{t_{i}}^{I}\right|}{\sum_{i=1}^{n} \omega_{i}} \cdot 100=\frac{1686,07}{470293,73} \cdot 100=0,358514241 \%
\end{gathered}
$$

- if the proceeding's structure which draws up the trend for $\omega$ variable, where $\omega=$ G.D.P. per capita in PPP for the United States of America, selects a quadratic exposure $\omega_{t_{i}}=a+b \cdot t_{i}+c t_{i}^{2}, a$ and $b$ will be [4]:

Table 11. The „radiography” for the values of the G.D.P. per capita in PPP,

\begin{tabular}{|c|c|c|c|c|c|c|c|c|}
\hline \multirow{2}{*}{ YEARS } & \multirow{2}{*}{$\begin{array}{l}\text { G.D.P. PER CAPITA IN PPP } \\
\text { THE UNITED STATES } \\
\text { (\$) } \\
\left(\omega_{i}\right)\end{array}$} & \multicolumn{7}{|c|}{ PARABOLIC TENDENCY } \\
\hline & & $t_{i}$ & $t_{i}^{2}$ & $t_{i}^{3}$ & $t_{i}^{4}$ & $t_{i}^{2} \omega_{i}$ & $\omega_{t_{i}}=a+b \xi_{i}+c \xi_{i}^{2}$ & $\left|\omega_{i}-\omega_{t_{i}}\right|$ \\
\hline 2010 & 49479,25 & -4 & 16 & -64 & 256 & 791668,00 & 49399,71139 & 79,54 \\
\hline 2011 & 49883,11 & -3 & 9 & -27 & 81 & 448947,00 & 49984,40968 & 101,30 \\
\hline 2012 & 50632,44 & -2 & 4 & -8 & 16 & 202529,76 & 50624,43165 & 8,01 \\
\hline 2013 & 51208,89 & -1 & 1 & -1 & 1 & 51208,89 & 51319,77729 & 110,89 \\
\hline 2014 & 52080,79 & 0 & 0 & 0 & 0 & 0 & 52070,44662 & 10,34 \\
\hline 2015 & 53187,57 & +1 & 1 & +1 & 1 & 53187,57 & 52876,43963 & 311,13 \\
\hline 2016 & 53631,76 & +2 & 4 & +8 & 16 & 214527,04 & 53737,75631 & 106,00 \\
\hline 2017 & 54470,80 & +3 & 9 & +27 & 81 & 490237,20 & 54654,39668 & 183,60 \\
\hline 2018 & 55719,12 & +4 & 16 & +64 & 256 & 891505,92 & 55626,36072 & 92,76 \\
\hline TOTAL & 470293,73 & 0 & 60 & 0 & 708 & 3143811,38 & & 1003,57 \\
\hline
\end{tabular}
in the United States of America, if this indicates a quadratic exposure

$$
\begin{gathered}
a=\frac{708 \cdot 470293,73-60 \cdot 3143811,38}{9 \cdot 708-60^{2}}=52070,44662 \quad b=\frac{46699,87}{60}=778,3311667 \\
c=\frac{9 \cdot 3143811,38-60 \cdot 470293,73}{9 \cdot 708-60^{2}}=27,66183983 \\
v_{I I}=\left[\frac{\sum_{i=1}^{n}\left|\omega_{i}-\omega_{t_{i}}^{I I}\right|}{n}: \frac{\sum_{i=1}^{n} \omega_{i}}{n}\right] \cdot 100=\frac{\sum_{i=1}^{n}\left|\omega_{i}-\omega_{t_{i}}^{I I}\right|}{\sum_{i=1}^{n} \omega_{i}} \cdot 100=\frac{1003,57}{470293,73} \cdot 100=0,21339217 \%
\end{gathered}
$$


- if the proceeding's structure which draws up the trend for $\omega$ variable, where $\omega=$ G.D.P. per capita in PPP for the United States of America, highlights an exponential leaning $\omega_{t_{i}}=a b^{t_{i}}, a$ and $b$ will be [4]:

Table 12. The "radiography" for the values of the G.D.P. per capita in PPP, in the United States of America, if this indicates an exponential exposure

\begin{tabular}{|c|c|c|c|c|c|c|c|}
\hline \multirow{2}{*}{ YEARS } & \multirow{2}{*}{$\begin{array}{c}\text { G.D.P. PER } \\
\text { CAPITA IN PPP } \\
\text { THE UNITED } \\
\text { STATES } \\
\text { (\$) } \\
\left(\omega_{i}\right)\end{array}$} & \multicolumn{6}{|c|}{ EXPONENTIAL TENDENCY } \\
\hline & & $t_{i}$ & $\lg \omega_{i}$ & $t_{i} \lg \omega_{i}$ & $\lg \omega_{t_{i}}=\lg a+t_{i} \lg b$ & $\omega_{t_{i}}=a b^{t_{i}}$ & $\left|\omega_{i}-\omega_{t_{i}}\right|$ \\
\hline 2010 & 49479,25 & -4 & 4,694423108 & $-18,77769243$ & 4,691976054 & 49201,24065 & 278,01 \\
\hline 2011 & 49883,11 & -3 & 4,697953522 & $-14,09386057$ & 4,698432827 & 49938,19341 & 55,08 \\
\hline 2012 & 50632,44 & -2 & 4,704428857 & $-9,408857713$ & 4,705117914 & 50712,83785 & 80,4 \\
\hline 2013 & 51208,89 & -1 & 4,709345362 & $-4,709345362$ & 4,711346373 & 51445,37923 & 236,49 \\
\hline 2014 & 52080,79 & 0 & 4,716677563 & 0 & 4,717803146 & 52215,94545 & 135,15 \\
\hline 2015 & 53187,57 & +1 & 4,725810149 & 4,725810149 & 4,724259919 & 52998,05347 & 189,52 \\
\hline 2016 & 53631,76 & +2 & 4,729422049 & 9,458844098 & 4,730716692 & 53791,87617 & 160,12 \\
\hline 2017 & 54470,80 & +3 & 4,736163754 & 14,20849126 & 4,737173465 & 54597,89010 & 127,09 \\
\hline 2018 & 55719,12 & +4 & 4,746004249 & 18,98401700 & 4,743630238 & 55415,37009 & 303,75 \\
\hline TOTAL & 470293,73 & 0 & 42,46022831 & 0,387406432 & & & 1565,61 \\
\hline
\end{tabular}

$$
\begin{gathered}
\lg a=\frac{42,46022831 \cdot 60}{9 \cdot 60}=4,717803146 \quad \lg b=\frac{9 \cdot 0,387406432}{9 \cdot 60}=0,006456773 \\
v_{\text {exp }}=\left[\frac{\sum_{i=1}^{n}\left|\omega_{i}-\omega_{t_{i}}^{\exp }\right|}{n}: \frac{\sum_{i=1}^{n} \omega_{i}}{n}\right] \cdot 100=\frac{\sum_{i=1}^{n}\left|\omega_{i}-\omega_{t_{i}}^{\exp }\right|}{\sum_{i=1}^{n} \omega_{i}} \cdot 100=\frac{1565,61}{470293,73} \cdot 100=0,33 \% \\
v_{I I}=0,21 \%<v_{\text {exp }}=0,33 \%<v_{I}=0,36 \%
\end{gathered}
$$

The values concerining the G.D.P. per capita in PPP for the United States of America, between 2010-2019, touch a quadratic "target” $\omega_{t_{i}}=a+b \cdot t_{i}+c t_{i}^{2}$

$$
\begin{aligned}
& \omega_{2021}^{G D P_{-} P E R_{-} C A P I T A_{-} P P P}=52070,44662+778,3311667 \cdot 7+27,66183983 \cdot 7^{2}=58874,19 \_\$ \\
& \omega_{2022_{-} P E R_{-} C A P I T A_{-} P P P}^{\text {GPP }}=52070,44662+778,3311667 \cdot 8+27,66183983 \cdot 8^{2}=60067,5_{-} \$
\end{aligned}
$$

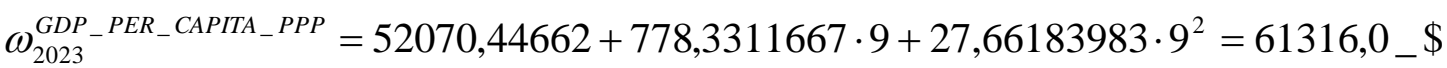

$$
\begin{aligned}
& \omega_{2024_{-} P E R_{-} C A P I T A_{-} P P P}^{G P P}=52070,44662+778,3311667 \cdot 10+27,66183983 \cdot 10^{2}=62619,9 \_\$ \\
& \omega_{2025}^{\text {GDP_PER }}{ }^{\text {CAPITA }}{ }^{P P P}=52070,44662+778,3311667 \cdot 11+27,66183983 \cdot 11^{2}=63979,2_{-} \$ \\
& \omega_{2026_{-}{ }^{\text {GDER }} \text { CAPITA_ }_{-} P P P}=52070,44662+778,3311667 \cdot 12+27,66183983 \cdot 12^{2}=65393,7 \_\$
\end{aligned}
$$

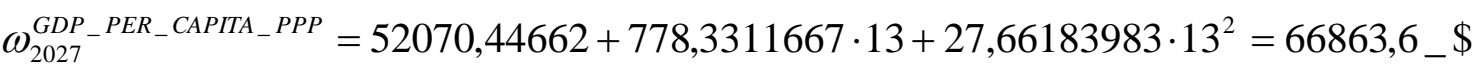

$$
\begin{aligned}
& \omega_{2028}^{G D P_{-} P E R_{-} C A P I T A_{-} P P P}=52070,44662+778,3311667 \cdot 14+27,66183983 \cdot 14^{2}=68388,8_{-} \$ \\
& \omega_{2029}^{\text {GDP } P E R_{-} C A P I T A_{-} P P P}=52070,44662+778,3311667 \cdot 15+27,66183983 \cdot 15^{2}=69969,3 \_\$ \\
& \omega_{2030_{-}{ }^{G D P R_{-} C A P I T A_{-} P P P}}=52070,44662+778,3311667 \cdot 16+27,66183983 \cdot 16^{2}=71605,2 \_\$
\end{aligned}
$$




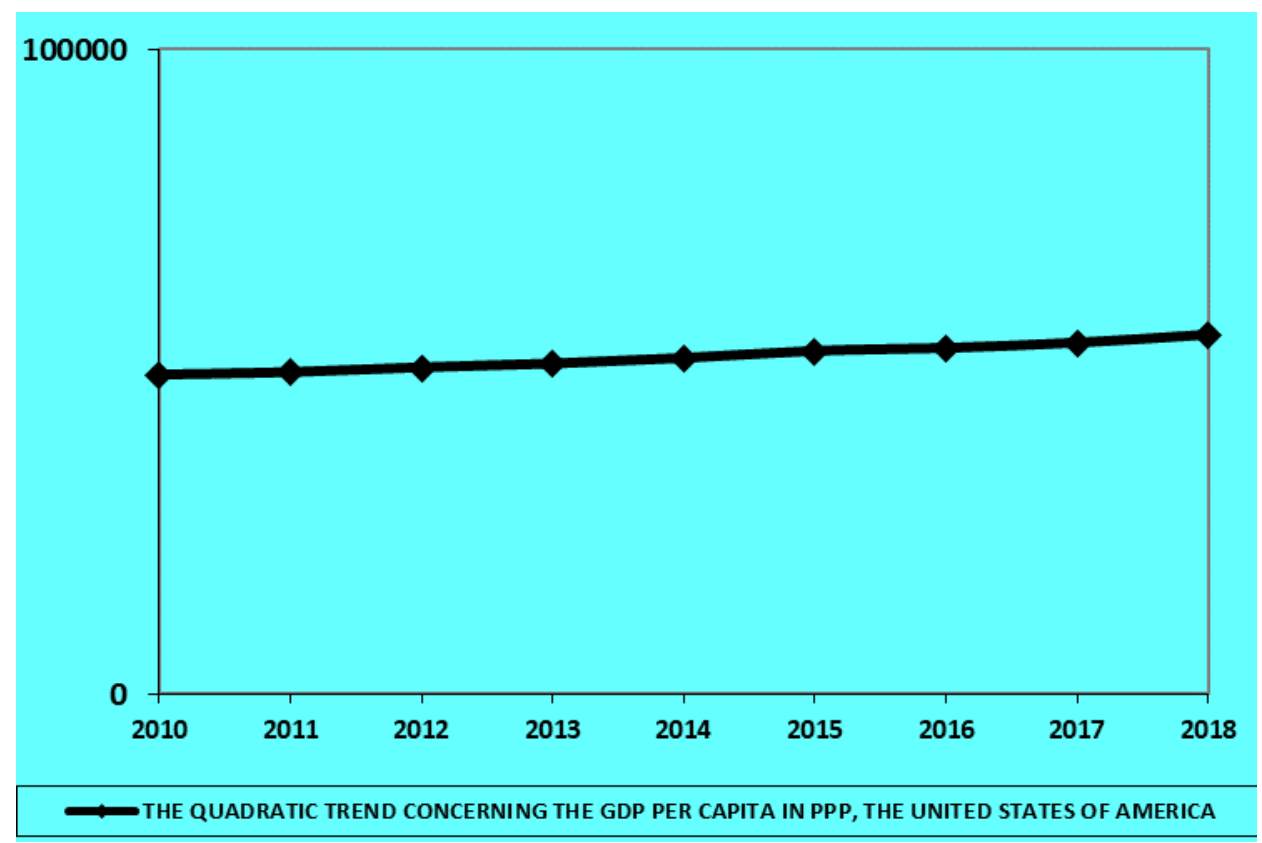

Graph 3 The quadratic „radiograhy” of the statistical data which show us the "wellness" of the G.D.P. per capita in PPP for the United States, between 2010-2019

4. The proceeding' s steps, which pursue the values regarding the G.D.P. per capita in PPS for the United States of America, for to achieve them wellness' diagnostic

Table 13. The G.D.P. per capita in PPS's configuration in the United States, between 2010-2018

\begin{tabular}{|c|c|}
\hline \multirow{2}{*}{ YEARS } & $\begin{array}{c}\text { G.D.P. PER CAPITA IN PPS } \\
\text { THE UNITED STATES OF AMERICA } \\
\text { Index (EU27_2020=100) }\left(\gamma_{i}\right)\end{array}$ \\
\hline 2010 & 147 \\
\hline 2011 & 145 \\
\hline 2012 & 148 \\
\hline 2013 & 147 \\
\hline 2014 & 148 \\
\hline 2016 & 149 \\
\hline 2017 & 143 \\
\hline 2018 & 140 \\
\hline & Source ${ }_{,}$EUUROSTAT” \\
\hline
\end{tabular}

- if the proceeding's structure which draws up the trend for $\gamma$ variable, where $\gamma=$ G.D.P. per capita in PPS for the United States of America, selects a linear exposure $\gamma_{t_{i}}=a+b \cdot t_{i}, a$ and $b$ will be [4]:

Table 14. The "radiography” for the values of the G.D.P. per capita in PPS, in the United States of America, if this indicates a linear exposure

\begin{tabular}{|c|c|c|c|c|c|c|}
\hline \multirow{2}{*}{ YEARS } & \multirow{2}{*}{$\begin{array}{l}\text { G.D.P. PER CAPITA } \\
\text { IN PPS } \\
\text { THE UNITED STATES } \\
\text { (\$) } \\
\left(\gamma_{i}\right)\end{array}$} & \multicolumn{5}{|c|}{ LINEAR TENDENCY } \\
\hline & & $t_{i}$ & $t_{i}^{2}$ & $t_{i} \omega_{i}$ & $\gamma_{t_{i}}=a+b t_{i}$ & $\left|\gamma_{i}-\gamma_{t_{i}}\right|$ \\
\hline 2010 & 147 & -4 & 16 & -588 & 148,1111110 & 1 \\
\hline 2011 & 145 & -3 & 9 & -435 & 147,5944444 & 3 \\
\hline 2012 & 148 & -2 & 4 & -296 & 146,8777777 & 1 \\
\hline 2013 & 147 & -1 & 1 & -147 & 146,1611111 & 1 \\
\hline 2014 & 148 & 0 & 0 & 0 & 145.4444444 & 3 \\
\hline 2015 & 149 & +1 & 1 & 149 & 144,7277777 & 4 \\
\hline 2016 & 143 & +2 & 4 & 286 & 144,0111111 & 1 \\
\hline 2017 & 140 & +3 & 9 & 420 & 143,2944444 & 3 \\
\hline 2018 & 142 & +4 & 16 & 568 & 142,5777777 & 1 \\
\hline TOTAL & 1309 & 0 & 60 & -43 & & 18 \\
\hline
\end{tabular}




$$
\begin{gathered}
a=\frac{1309,73 \cdot 60}{9 \cdot 60}=145,4444444 \quad b=\frac{9 \cdot(-43)}{9 \cdot 60}=-0,716666666 \\
v_{I}=\left[\frac{\sum_{i=1}^{n}\left|\gamma_{i}-\gamma_{t_{i}}^{I}\right|}{n}: \frac{\sum_{i=1}^{n} \gamma_{i}}{n}\right] \cdot 100=\frac{\sum_{i=1}^{n}\left|\gamma_{i}-\gamma_{t_{i}}^{I}\right|}{\sum_{i=1}^{n} \gamma_{i}} \cdot 100=\frac{18}{1309} \cdot 100=1,37 \%
\end{gathered}
$$

- if the proceeding's structure which draws up the trend for $\gamma$ variable, where $\gamma=$ G.D.P. per capita in PPS for the United States of America, selects a quadratic exposure $\gamma_{t_{i}}=a+b \cdot t_{i}+c t_{i}^{2}, a$ and $b$ will be [4]:

Table 15. The „radiography” for the values of the G.D.P. per capita in PPS,

\begin{tabular}{|c|c|c|c|c|c|c|c|c|}
\hline \multirow{2}{*}{ YEARS } & \multirow{2}{*}{$\begin{array}{c}\text { G.D.P. PER CAPITA } \\
\text { IN PPS } \\
\text { THE UNITED } \\
\text { STATES } \\
\left(\gamma_{i}\right)\end{array}$} & \multicolumn{7}{|c|}{ PARABOLIC TENDENCY } \\
\hline & & $t_{i}$ & $t_{i}^{2}$ & $t_{i}^{3}$ & $t_{i}^{4}$ & $t_{i}^{2} \gamma_{i}$ & $\gamma_{t_{i}}=a+b \gamma_{i}+c \gamma_{i}^{2}$ & $\left|\gamma_{i}-\gamma_{t_{i}}\right|$ \\
\hline 2010 & 147 & -4 & 16 & -64 & 256 & 2352 & 145,9575757 & 1 \\
\hline 2011 & 145 & -3 & 9 & -27 & 81 & 1305 & 147,0060606 & 2 \\
\hline 2012 & 148 & -2 & 4 & -8 & 16 & 592 & 147,5502164 & 1 \\
\hline 2013 & 147 & -1 & 1 & -1 & 1 & 147 & 147,5900433 & 1 \\
\hline 2014 & 148 & 0 & 0 & 0 & 0 & 0 & 147,1255411 & 1 \\
\hline 2015 & 149 & +1 & 1 & +1 & 1 & 149 & 146,1567099 & 3 \\
\hline 2016 & 143 & +2 & 4 & +8 & 16 & 572 & 144,6835498 & 2 \\
\hline 2017 & 140 & +3 & 9 & +27 & 81 & 1260 & 142,7060606 & 3 \\
\hline 2018 & 142 & +4 & 16 & +64 & 256 & 2272 & 140,2242424 & 2 \\
\hline TOTAL & 1309 & 0 & 60 & 0 & 708 & 8649 & & 16 \\
\hline
\end{tabular}
in the United States of America, if this indicates a quadratic exposure

$$
\begin{gathered}
a=\frac{708 \cdot 1309-60 \cdot 8649}{9 \cdot 708-60^{2}}=147,1255411 \quad b=-\frac{43}{60}=-0,716666666 \\
c=\frac{9 \cdot 8649-60 \cdot 1309}{9 \cdot 708-60^{2}}=-0,252164502 \\
v_{I I}=\left[\frac{\sum_{i=1}^{n}\left|\gamma_{i}-\gamma_{t_{i}}^{I I}\right|}{n}: \frac{\sum_{i=1}^{n} \gamma_{i}}{n}\right] \cdot 100=\frac{\sum_{i=1}^{n}\left|\gamma_{i}-\gamma_{t_{i}}^{I I}\right|}{\sum_{i=1}^{n} \gamma_{i}} \cdot 100=\frac{16}{1309} \cdot 100=1,22 \%
\end{gathered}
$$

\begin{tabular}{|c|c|c|c|c|c|c|c|}
\hline \multirow{2}{*}{ YEARS } & \multirow{2}{*}{$\begin{array}{c}\text { G.D.P. PER } \\
\text { CAPITA } \\
\text { IN PPS } \\
\text { THE UNITED } \\
\text { STATES } \\
\left(\gamma_{i}\right)\end{array}$} & \multicolumn{6}{|c|}{ EXPONENTIAL TENDENCY } \\
\hline & & $t_{i}$ & $\lg \gamma_{i}$ & $t_{i} \lg \gamma_{i}$ & $\lg \gamma_{t_{i}}=\lg a+t_{i} \lg b$ & $\gamma_{t_{i}}=a b^{t_{i}}$ & $\left|\gamma_{i}-\gamma_{t_{i}}\right|$ \\
\hline 2010 & 147 & -4 & 2,167317335 & $-8,669269339$ & 2,171261751 & 148,3411874 & 1 \\
\hline 2011 & 145 & -3 & 2,161368002 & $-6,484104007$ & 2,169098113 & 147,6039953 & 3 \\
\hline 2012 & 148 & -2 & 2,170261715 & $-4,340523431$ & 2,166934475 & 146,8704667 & 1 \\
\hline 2013 & 147 & -1 & 2,167317335 & $-2,167317335$ & 2,164770837 & 146,1405835 & 1 \\
\hline 2014 & 148 & 0 & 2,170261715 & 0 & 2,162607199 & 145,4143275 & 3 \\
\hline 2015 & 149 & +1 & 2,173186268 & 2,173186268 & 2,160443561 & 144,6916806 & 4 \\
\hline 2016 & 143 & +2 & 2,155336037 & 4,310672075 & 2,158279923 & 143,9726250 & 1 \\
\hline 2017 & 140 & +3 & 2,146128036 & 6,438384107 & 2,156116285 & 143,2571428 & 3 \\
\hline 2018 & 142 & +4 & 2,152288344 & 8,609153378 & 2,153952647 & 142,5452162 & 0 \\
\hline TOTAL & 1309 & 0 & 19,46346479 & $-0,129818284$ & & & 17 \\
\hline
\end{tabular}

- if the proceeding's structure which draws up the trend for $\gamma$ variable, where $\gamma=$ G.D.P. per capita in PPS for the United States of America, selects an exponential exposure $\gamma_{t_{i}}=a b^{t_{i}}, a$ and $b$ will be [4]:

Table 16. The „radiography” for the values of the G.D.P. per capita in PPS, in the United States of America, if this indicates an exponential exposure

$\lg a=\frac{19,4346479 \cdot 60}{9 \cdot 60}=2,162607199 \quad \lg b=\frac{9 \cdot(-0,129818284)}{9 \cdot 60}=-0,002163638$ 


$$
\begin{aligned}
& v_{\text {exp }}=\left[\frac{\sum_{i=1}^{n}\left|\gamma_{i}-\gamma_{t_{i}}^{\exp }\right|}{n}: \frac{\sum_{i=1}^{n} \gamma_{i}}{n}\right] \cdot 100=\frac{\sum_{i=1}^{n}\left|\gamma_{i}-\gamma_{t_{i}}^{\exp }\right|}{\sum_{i=1}^{n} \lambda_{i}} \cdot 100=\frac{17}{1309} \cdot 100=1,30 \% \\
& v_{I I}=1,22 \%<v_{I}=1,37 \%<v_{\text {exp }}=1,30 \%
\end{aligned}
$$

The values regarding the G.D.P. per capita in PPS for the United States of America, in the period 20102019 , hit a quadratic "target” $\gamma_{t_{i}}=a+b \cdot t_{i}+c t_{i}^{2}$

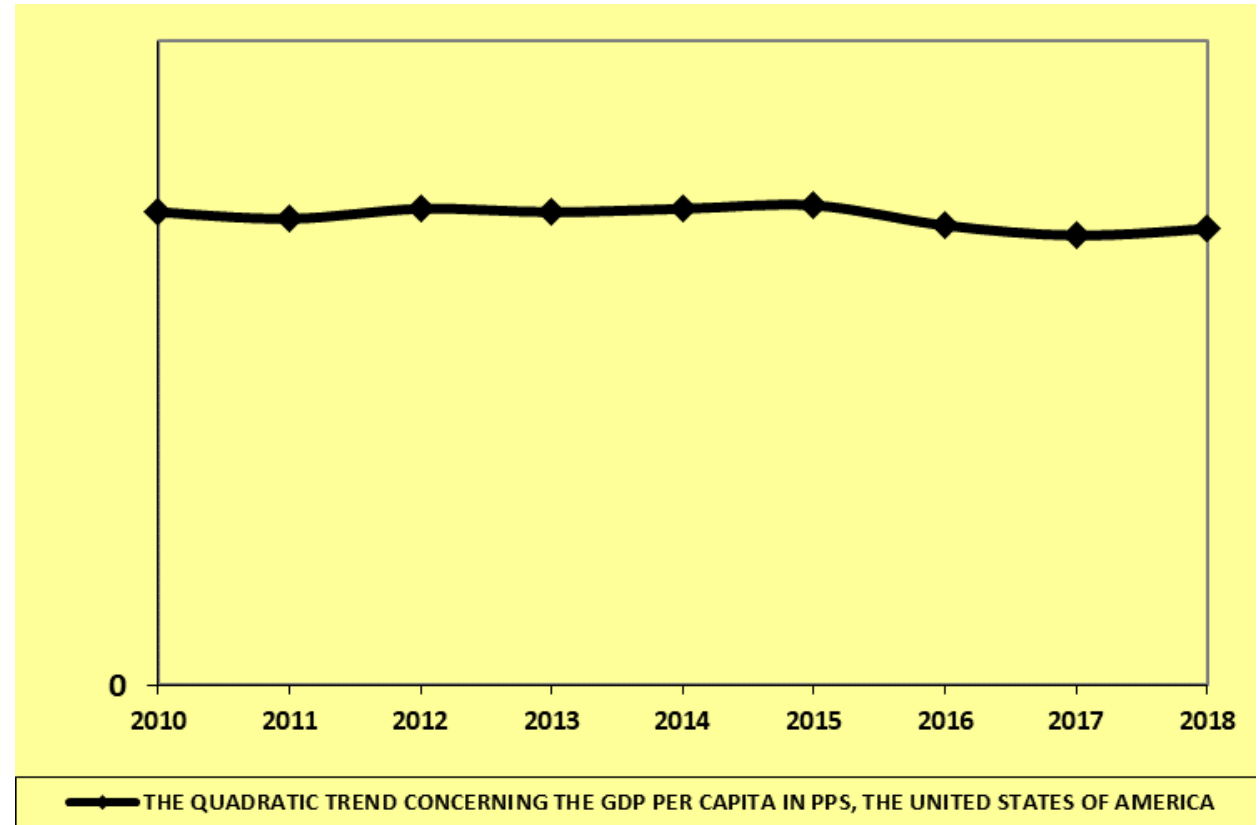

Graph 4 The quadratic „radiography” of the statistical data which show us the „wellness" of the G.D.P. per capita in PPS for the United States, between 2010-2019

$$
\gamma_{2021}^{\text {GDP } P_{-} P E R_{-} C A P I T A_{-} P P S}=147,1255411+(-0,716666666) \cdot 7+(-0,252164502) \cdot 7^{2}=129,8
$$

\section{Conclusions}

We can see in the period 2021-2030, rises concerning the United States of America's real G.D.P., from $\$ 19164,5$ billions in 2021, to $\$ 20368$,9 billions in 2030. Alike, we can observe between 2021-2030, increases in relative sizes, regarding the real G.D.P.'s annual growth for the United States of America, from 2,8 \% in 2021 , to $4,3 \%$ in 2030. Also, we can remark in the period 2021-2030, augmentations concerning the United States of America's G.D.P. per capita in PPP, from \$58874,19 in 2021, to \$71605,2 in 2030.

\section{References}

1. Fioramonti, L. (2013). Gross Domestic Problem: The Politics Behind the World's Most Powerful Number, Zed Books Publishing House, London.

2. Fioramonti, L. (2017). Wellbeing Economy - Success in a World Without Growth, Pan Macmillan Publishing House, London.

3. Fleurbaey, M. (2013). Beyond GDP: Measuring Welfare and Assessing Sustainability, Oxford University Press Publishing House, London, 2013.

4. Gauss, C.F. (1986). Disquisitiones Arithmeticae and other papers on number theory, english translation Springer Publishing House, New-York, 1986.

5. Hoekstra, R. (2019). Replacing GDP by 2030: Towards a Common Language for the Well-Being and Sustainability Community, Cambridge University Press Publishing House, London, 2019. 